Stephen J. Freedland, Duke University, Durham; Ronald C. Chen, University of North Carolina, Chapel Hill, NC; R. Bryan Rumble, American Society of Clinical Oncology, Alexandria, VA; Antonio Finelli, Princess Margaret Hospital, University Health Network, Toronto; Colin Wackett, Patient Advocate, Orillia, Ontario, Canada; Susan Slovin, Memorial Sloan Kettering Cancer Center, New York, NY; Mark N. Stein, Rutgers Cancer Institute of New Jersey, New Brunswick, NJ; David S. Mendelson, Pinnacle Oncology Hematology, Scottsdale, AZ; Howard M. Sandler, Cedars-Sinai Medical Center, Los Angeles, CA.

Published online ahead of print at www.jco.org on November 3, 2014.

Processed as a Rapid Communication manuscript.

Editor's note: This American Society of Clinical Oncology clinical practice guideline endorsement provides recommendations based on the review and

analysis of the relevant literature in the adjuvant and salvage radiotherapy after prostatectomy guideline. Additional information, which may include a methodology supplement, data supplements, slide sets, patient versions, frequently asked questions, and other clinical tools and resources, are available at www.asco.org/endorsements/ adjuvantRTprostatectomy.

Authors' disclosures of potential conflicts of interest are found in the article online at www.jco.org. Author contributions are found at the end of this article.

Corresponding author: American Society of Clinical Oncology, 2318 Mill Rd, Suite 800, Alexandria, VA 22314;

e-mail: guidelines@asco.org.

(๑) 2014 by American Society of Clinical Oncology

$0732-183 \times / 14 / 3299-1 / \$ 20.00$

DOI: $10.1200 / J C O .2014 .58 .8525$

\title{
Adjuvant and Salvage Radiotherapy After Prostatectomy: American Society of Clinical Oncology Clinical Practice Guideline Endorsement
}

Stephen J. Freedland, R. Bryan Rumble, Antonio Finelli, Ronald C. Chen, Susan Slovin, Mark N. Stein, David S. Mendelson, Colin Wackett, and Howard M. Sandler

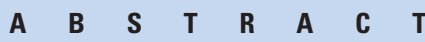

\section{Purpose}

To endorse the American Urological Association (AUA)/American Society for Radiation Oncology (ASTRO) guideline on adjuvant and salvage radiotherapy after prostatectomy. The American Society of Clinical Oncology (ASCO) has a policy and set of procedures for endorsing clinical practice guidelines developed by other professional organizations.

\section{Methods}

The guideline on adjuvant and salvage radiotherapy after prostatectomy was reviewed for developmental rigor by methodologists. An ASCO endorsement panel then reviewed the content and recommendations.

\section{Results}

The panel determined that the guideline recommendations on adjuvant and salvage radiotherapy after prostatectomy, published in August 2013, are clear, thorough, and based on the most relevant scientific evidence. ASCO endorsed the guideline on adjuvant and salvage radiotherapy after prostatectomy, adding one qualifying statement that not all candidates for adjuvant or salvage radiotherapy have the same risk of recurrence or disease progression, and thus, risk-benefit ratios are not the same for all men. Those at the highest risk for recurrence after radical prostatectomy include men with seminal vesicle invasion, Gleason score 8 to 10, extensive positive margins, and detectable postoperative prostate-specific antigen (PSA).

\section{Recommendations}

Physicians should discuss adjuvant radiotherapy with patients with adverse pathologic findings at prostatectomy (ie, seminal vesicle invasion, positive surgical margins, extraprostatic extension) and salvage radiotherapy with patients with PSA or local recurrence after prostatectomy. The discussion of radiotherapy should include possible short- and long-term adverse effects and potential benefits. The decision to administer radiotherapy should be made by the patient and multidisciplinary treatment team, keeping in mind that not all men are at equal risk of recurrence or clinically meaningful disease progression. Thus, the risk-benefit ratio will differ for each patient.

\section{J Clin Oncol 32. (C) 2014 by American Society of Clinical Oncology}

\section{INTRODUCTION}

In 2014, an estimated 233,000 men in the United States will be diagnosed with prostate cancer, ${ }^{1}$ and an estimated 29,480 will die as a result of it. ${ }^{1}$ For men eligible for surgery with curative intent, radical prostatectomy is an option; however, approximately one third of all patients treated this way will experience recurrence within a decade. ${ }^{2}$ Risk for recurrence after radical prostatectomy is greater for men with any of the following: positive surgical margins, seminal vesicle invasion, extraprostatic extension, higher Gleason score, and detectable postoperative prostate-specific antigen (PSA). ${ }^{2,3}$ The purpose of this American Society of Clinical Oncology (ASCO) endorsement is to support the American Urological Association (AUA)/American Society for Radiation Oncology (ASTRO) guideline on adjuvant and salvage radiotherapy after prostatectomy by Thompson et $\mathrm{al}^{2}$, published in August 2013 in Journal of Urology. This ASCO endorsement reinforces the recommendations offered in the AUA/ASTRO guideline and acknowledges the effort put forth by the AUA/ASTRO to produce an evidencebased guideline informing practitioners who care for patients being considered for adjuvant and 


\section{ASCO Endorses the Adjuvant and Salvage Radiotherapy After Prostatectomy: AUA/ASTRO Clinical Practice Guideline, With Qualifying Statements Developed by the Endorsement Panel}

\section{Guideline Questions}

The four principal questions involved:

1) Patient counseling

2) Use of radiotherapy in the adjuvant and salvage contexts

3) Definition of biochemical recurrence

4) Conduct of a restaging evaluation

\section{Target Population}

- Men offered prostate cancer treatment with prostatectomy

\section{Target Audience}

- Primary care providers, radiation and medical oncologists, urologists, and other providers

\section{Methods}

- An ASCO Endorsement Panel was convened to consider endorsing the Adjuvant and Salvage Radiotherapy After Prostatectomy: AUA/ASTRO Clinical Practice Guideline recommendations, which were based on a systematic review of the medical literature. The ASCO Endorsement Panel evaluated the methodology employed in the AUA/ASTRO guideline using the AGREE II review instrument. The ASCO Endorsement Panel carefully reviewed the AUA/ASTRO guideline content to determine appropriateness for ASCO endorsement.

\section{ASCO Key Recommendations for Adjuvant and Salvage Radiotherapy After Prostatectomy: ASCO Qualifying} Statements (in bold italics)

1. Patients who are being considered for management of localized prostate cancer with radical prostatectomy should be informed of the potential for adverse pathologic findings that portend a higher risk of cancer recurrence and that these findings may suggest a potential benefit of additional therapy after surgery.

2. Patients with adverse pathologic findings, including seminal vesicle invasion, positive surgical margins, and extraprostatic extension, should be informed that adjuvant radiotherapy, compared with radical prostatectomy only, reduces the risk of biochemical prostate-specific antigen (PSA) recurrence, local recurrence, and clinical progression of cancer. They should also be informed that the impact of adjuvant radiotherapy on subsequent metastases and overall survival is less clear; one of two randomized controlled trials that addressed these outcomes indicated a benefit, but the other trial did not demonstrate a benefit (reduced risk of metastasis and death).

3. Physicians should offer* adjuvant radiotherapy to patients with adverse pathologic findings at prostatectomy, including seminal vesicle invasion, positive surgical margins, or extraprostatic extension, because of demonstrated reductions in biochemical recurrence, local recurrence, and clinical progression.

4. Patients should be informed that the development of a PSA recurrence after surgery is associated with a higher risk of development of metastatic prostate cancer or death resulting from the disease. $\dagger$ Congruent with this clinical principle, physicians should regularly monitor PSA after radical prostatectomy to enable early administration of salvage therapies if appropriate.

5. Clinicians should define biochemical recurrence as a detectable or increasing PSA value after surgery that is $>0.2 \mathrm{ng} /$ $\mathrm{mL}$, with a second confirmatory level $>0.2 \mathrm{ng} / \mathrm{mL}$.

6. A restaging evaluation $\$$ in a patient with a PSA recurrence may be considered.

(continued on following page) 


\section{THE BOTTOM LINE (CONTINUED)}

7. Physicians should offer ${ }^{\star}$ salvage radiotherapy to patients with PSA or local recurrence after radical prostatectomy in whom there is no evidence of distant metastatic disease.

8. Patients should be informed that the effectiveness of radiotherapy for PSA recurrence\| is greatest when administered at lower levels of PSA.

9. Patients should be informed of the possible short- and long-term urinary, bowel, and sexual adverse effects of radiotherapy as well as of the potential benefits of controlling disease recurrence. $\mathbf{f}$

\section{Qualifying Statements}

${ }^{\star}$ The word "offer" should be interpreted as having a detailed discussion with the patient about the risks and benefits of adjuvant radiotherapy. This discussion should be heavily influenced by our additional qualifying statementg and include a thorough discussion of the absolute risk of recurrence in light of exact pathologic findings and postoperative PSA levels. As such, although adjuvant ratiotherapy seems to be equally effective for all stages and postoperative PSA levels in terms of reducing the relative risk of recurrence, the absolute benefit is influenced by the absolute risk of recurrence. For men at low risk of recurrence, the benefits are modest. Moreover, all potential benefits must be weighed against the known risks.

$\dagger$ This Endorsement Panel concurs, as noted in the discussion for Recommendation 3 and in our qualifying statement, 9 that not all men are at equal risk of recurrence. However, at this time, there are insufficient data to recommend different follow-up strategies based on differing risk of recurrence, and thus, all men should be observed after surgery.

$\ddagger$ Defining an exact cut point for PSA recurrence is challenging. We acknowledge this difficulty, and although we agree that 0.2 $\mathrm{ng} / \mathrm{mL}$ is a reasonable cutoff and is widely used in research publications and in clinical practice, the benefits of using this cut point versus others are unclear. As such, we believe that the evidence to support this recommendation was clinical practice rather than clinical evidence.

$\S$ Although the endorsement panel agrees with this statement, the discussion centered on which imaging modalities to use. At this time, there is no clear consensus, and unfortunately, all imaging modalities have limited sensitivity and specificity in the low PSA range, where salvage radiotherapy is most effective (ie, PSA $<1 \mathrm{ng} / \mathrm{mL}$ ). We note that this is a rapidly evolving field with much research being performed, and it is hoped that in the future, there will be more clarity on this point.

$\|$ Although PSA control rates are best when salvage radiotherapy is administered when the PSA is $<1 \mathrm{ng} / \mathrm{mL}$, there is no guarantee of cure, because salvage radiotherapy is not curative for all men. There are many technical issues related to measuring the rate of PSA increase, typically measured as PSA doubling time (PSADT; ie, when to start counting, how many values, over what time period, and so on), but data suggest a short PSADT predicts a poor response to salvage radiotherapy in terms of PSA control. However, because these men are at the highest risk of clinically meaningful progression, they stand to gain the most from salvage radiotherapy, and indeed, retrospective data suggest that salvage radiotherapy improves overall survival, albeit only in those with a short PSADT.

INot all men who are candidates for adjuvant radiotherapy have the same risk of recurrence, and although radiotherapy is equally effective in terms of relative risk reduction, not all men will derive the same benefit. Items such as Gleason score (especially Gleason score 8 to 10), pathologic findings (especially seminal vesicle invasion or extensive positive margins), and an elevated postoperative PSA place men at particularly high risk of recurrence or clinical progression, and these men likely derive the greatest benefit in terms of absolute risk reduction from adjuvant radiotherapy. For other men, the absolute risk benefit is likely lower, resulting in a less favorable risk-benefit ratio. Similarly, not all men who develop a PSA recurrence have the same risk of clinically meaningful disease progression, and therefore, the risk-benefit ratio for salvage radiotherapy is likewise different for each patient.

\section{Additional Resources}

More information, including a Data Supplement, a Methodology Supplement, slide sets, and clinical tools and resources, is available at www.asco.org/endorsements/adjuvantRTprostatectomy. Patient information is available at www.cancer.net. A link to the Adjuvant and Salvage Radiotherapy After Prostatectomy: AUA/ASTRO Clinical Practice Guideline can be found at http://www.auanet.org/ education/guidelines/radiation-after-prostatectomy.cfm. ASCO believes that cancer clinical trials are vital to inform medical decisions and improve cancer care, and that all patients should have the opportunity to participate.

salvage radiotherapy after prostatectomy. The issues addressed in the original guideline as well as this endorsement are the use of radiotherapy in the adjuvant and salvage settings, definition of biochemical recurrence, conduct of a restaging evaluation, and role of patient counseling. Although we are, overall, endorsing the recommendations, we believed it was important to clarify certain aspects of the guideline as well as add a qualifying statement to help put these guidelines into clinical context. 
OVERVIEW OF ASCO GUIDELINE ENDORSEMENT PROCESS

ASCO has policies and procedures for endorsing practice guidelines that have been developed by other professional organizations. The goal of guideline endorsement is to increase the number of highquality, ASCO-vetted guidelines available to the ASCO membership. The ASCO endorsement process involves an assessment by ASCO staff of candidate guidelines for methodologic quality using the Rigour of Development subscale of the Appraisal of Guidelines for Research and Evaluation II (AGREE II) instrument (details provided in Methodology Supplement).

\section{Disclaimer}

The clinical practice guideline and other guidance published herein are provided by ASCO to assist providers in clinical decision making. The information herein should not be relied on as being complete or accurate, nor should it be considered as inclusive of all proper treatments or methods of care or as a statement of the standard of care. With the rapid development of scientific knowledge, new evidence may emerge between the time information is developed and when it is published or read. The information is not continually updated and may not reflect the most recent evidence. The information addresses only the topics specifically identified herein and is not applicable to other interventions, diseases, or stages of diseases. This information does not mandate any particular course of medical care. Furthermore, the information is not intended to substitute for the independent professional judgment of the treating provider, because the information does not account for individual variation among patients. For each recommendation, high, moderate, or low confidence that the recommendation reflects the net effect of a given course of action is indicated. The use of words like must, must not, should, and should not indicates that a course of action is recommended or not recommended for either most or many patients, but there is latitude for the treating physician to select other courses of action in individual cases. In all cases, the selected course of action should be considered by the treating provider in the context of treating the individual patient. Use of the information is voluntary. ASCO provides this information on an as-is basis and makes no warranty, express or implied, regarding the information. ASCO specifically disclaims any warranties of merchantability or fitness for a particular use or purpose. ASCO assumes no responsibility for any injury or damage to persons or property arising out of or related to any use of this information or for any errors or omissions.

\section{Guideline and Conflicts of Interest}

The endorsement panel was assembled in accordance with the ASCO Conflicts of Interest Management Procedures for Clinical Practice Guidelines (summarized at www.asco.org/rwc). Members of the endorsement panel completed the ASCO disclosure form, which requires disclosure of financial and other interests that are relevant to the subject matter of the guideline, including relationships with commercial entities that are reasonably likely to experience direct regulatory or commercial impact as the result of promulgation of the guideline. Categories for disclosure include employment relationships, consulting arrangements, stock ownership, honoraria, research funding, and expert testimony. In accordance with these procedures, the majority of the members of the endorsement panel did not disclose any such relationships.

\section{Clinical Questions and Target Population}

The AUA/ASTRO guideline addressed four principle questions regarding (1) patient counseling, (2) use of radiotherapy in the adjuvant and salvage contexts, (3) definition of biochemical recurrence, and (4) conduct of a restaging evaluation. The clinical questions and corresponding recommendations are listed in Table 1.

The target population for the AUA/ASTRO guideline is male patients with prostate cancer who were previously treated with radical prostatectomy and are being considered for adjuvant or salvage treatment with radiotherapy.

\section{Summary of Adjuvant and Salvage Radiotherapy After Prostatectomy Guideline Development Methodology}

The guideline was developed by the prostate guidelines panel of the AUA/ASTRO and included urologists, radiation oncologists, and a medical oncologist. The systematic literature review included a search of the PubMed, EMBASE, and Cochrane Collaboration databases from January 1, 1990, to December 15, 2012. Articles were eligible for inclusion if they reported on the use of radiotherapy after prostatectomy (if PSA data were available for at least $75 \%$ of all patients), quality-of-life outcomes, adverse effect outcomes, or imaging strategies to determine appropriateness of radiotherapy when recurrence is suspected. Preclinical studies, commentaries, editorials, and review articles were excluded. The searches identified 294 studies eligible for inclusion in the guideline.

The recommendations formed were deemed to be either: standards, recommendations, options, clinical principles, or expert opinions, depending on the quality and quantity of supporting evidence. Once recommendations were drafted, the guideline was subjected to a rigorous external review process, where the document was distributed to 75 peer reviewers (returned, $\mathrm{n}=44$; response rate, $59 \%$ ). All submitted comments were reviewed, and the draft was revised as needed. The guideline was then submitted to the governing body of each organization and then to the AUA and ASTRO Boards of Directors for final approval.

\section{RESULTS OF THE ASCO METHODOLOGY REVIEW}

The methodology review of the AUA/ASTRO guideline was completed independently by two ASCO guideline staff members using the Rigour of Development subscale from the AGREE II instrument. Detailed results of the scoring for this guideline are available on request to guidelines@asco.org. Overall, the adjuvant and salvage radiotherapy after prostatectomy guideline itself scored an acceptable 66\%. Although this guideline was well reported overall, no information was provided regarding plans for future updating, decreasing the score in the Rigour of Development domain. The preliminary ASCO content reviewers of the adjuvant and salvage radiotherapy after prostatectomy guideline, as well as the ASCO endorsement panel, found the recommendations well supported in the original guideline. Each section, including introduction, methods, background, and results sections, was clear and well referenced from the systematic review.

This is the most recent information as of the publication date. To view updates and the most recent information and to submit new evidence, please visit www.asco.org/endorsements/adjuvantRT prostatectomy or the ASCO guidelines wiki at www.asco.org/ guidelineswiki. 
Table 1. Adjuvant and Salvage Radiotherapy After Prostatectomy: AUA/ASTRO Research Questions and Recommendations*
Research question domains
1. Patient counseling
2. Use of radiotherapy in adjuvant and salvage contexts
3. Definition of biochemical recurrence
4. Conduct of restaging evaluation
Guideline recommendations

1. Patients who are being considered for management of localized prostate cancer with radical prostatectomy should be informed of potential for adverse pathologic findings that portend higher risk of cancer recurrence and that these findings may suggest potential benefit of additional therapy after surgery (clinical principle)

2. Patients with adverse pathologic findings, including seminal vesicle invasion, positive surgical margins, and extraprostatic extension, should be informed that adjuvant radiotherapy, compared with radical prostatectomy only, reduces risk of biochemical (PSA) recurrence, local recurrence, and clinical progression of cancer. They should also be informed that impact of adjuvant radiotherapy on subsequent metastases and overall survival is less clear; one of two randomized controlled trials that addressed these outcomes indicated benefit, but the other trial did not demonstrate a benefit (clinical principle)

3. Physicians should offer adjuvant radiotherapy to patients with adverse pathologic findings at prostatectomy, including seminal vesicle invasion, positive surgical margins, or extraprostatic extension, because of demonstrated reductions in biochemical recurrence, local recurrence, and clinical progression (standard; evidence strength: grade A)

4. Patients should be informed that development of PSA recurrence after surgery is associated with higher risk of development of metastatic prostate cancer or death resulting from disease; congruent with this clinical principle, physicians should regularly monitor PSA after radical prostatectomy to enable early administration of salvage therapies if appropriate (clinical principle)

5. Clinicians should define biochemical recurrence as detectable or rising PSA value after surgery that is $>0.2 \mathrm{ng} / \mathrm{mL}$, with second confirmatory level $>0.2$ $\mathrm{ng} / \mathrm{mL}$ (recommendation; evidence strength: grade C)

6. Restaging evaluation in patient with PSA recurrence may be considered (option; evidence strength: grade C)

7. Physicians should offer salvage radiotherapy to patients with PSA or local recurrence after radical prostatectomy, in whom there is no evidence of distant metastatic disease (recommendation; evidence strength: grade C)

8. Patients should be informed that effectiveness of radiotherapy for PSA recurrence is greatest when administered at lower levels of PSA (clinical principle)

9. Patients should be informed of possible short- and long-term urinary, bowel, and sexual side effects of radiotherapy as well as of potential benefits of controlling disease recurrence (clinical principle)

Abbreviations: ASTRO, American Society for Radiation Oncology; AUA, American Urological Association; PSA, prostate-specific antigen.

*Data adapted. ${ }^{2}$

\section{METHODS AND RESULTS OF ASCO UPDATED LITERATURE REVIEW}

ASCO guidelines staff updated the adjuvant and salvage radiotherapy after prostatectomy guideline literature search, using the same PubMed search strategy that was used in the original guideline. The search was restricted to articles published in English and to systematic reviews, meta-analyses, and randomized controlled trials.

The updated search yielded 319 records. A review of these results by the methodology team resulted in 16 abstracts being reviewed by the panel cochairs, which revealed one recently published article ${ }^{4}$ that supported a qualifying statement.

\section{RESULTS OF ASCO CONTENT REVIEW}

The ASCO endorsement panel (members listed in Table 2) reviewed the adjuvant and salvage radiotherapy after prostatectomy guideline and concurs that the recommendations are clear, thorough, and based on the most relevant scientific evidence in this content area and present options that will be acceptable to patients. Overall, the ASCO endorsement panel agrees with the recommendations as stated in the guideline, with minor clarifications noted, in addition to one qualifying statement.

\section{DISCUSSION}

The ASCO endorsement panel wants to highlight and qualify some of the statements from the adjuvant and salvage radiotherapy after prostatectomy guideline regarding better clarifying the risks and benefits and more clearly discussing what is meant by the word offer. Although
Recommendations 1 and 9 were accepted as is, qualifying statements were added for the rest as follows:

\section{Recommendation 2}

In the final sentence of the guideline statement, the word benefit is meant to imply reduced risk of metastasis and death.

Table 2. Adjuvant and Salvage Radiotherapy After Prostatectomy ASCO Endorsement Panel

\begin{tabular}{|c|c|}
\hline Name & Affiliation/Institution \\
\hline $\begin{array}{l}\text { Stephen J. Freedland, } \\
\text { MD (co-chair) }\end{array}$ & Duke University, Durham, NC \\
\hline $\begin{array}{l}\text { Howard M. Sandler, } \\
\text { MD, MS } \\
\text { (co-chair) }\end{array}$ & Cedars-Sinai Medical Center, Los Angeles, CA \\
\hline $\begin{array}{l}\text { Antonio Finelli, MD, } \\
\text { MSc }\end{array}$ & $\begin{array}{l}\text { Princess Margaret Hospital, University Health } \\
\text { Network, Toronto, Ontario, Canada }\end{array}$ \\
\hline $\begin{array}{l}\text { Ronald C. Chen, MD, } \\
\text { MPH }\end{array}$ & University of North Carolina, Chapel Hill, NC \\
\hline $\begin{array}{l}\text { Susan Slovin, MD, } \\
\text { PhD }\end{array}$ & $\begin{array}{l}\text { Memorial Sloan Kettering Cancer Center, New } \\
\text { York, NY }\end{array}$ \\
\hline Mark N. Stein, MD & $\begin{array}{l}\text { Rutgers Cancer Institute of New Jersey, New } \\
\text { Brunswick, NJ }\end{array}$ \\
\hline $\begin{array}{l}\text { David S. Mendelson, } \\
\text { MD }\end{array}$ & $\begin{array}{l}\text { Pinnacle Oncology Hematology, Scottsdale, AZ } \\
\text { PGIN representative }\end{array}$ \\
\hline Colin Wackett & Patient representative \\
\hline
\end{tabular}

Abbreviations: ASCO, American Society of Clinical Oncology; PGIN, Practice Guidelines Implementation Network. 


\section{Recommendation 3}

In the first sentence, the word offer should be interpreted as having a detailed discussion with the patient about the risks and benefits of adjuvant radiotherapy. This discussion should be heavily influenced by our additional qualifying statement and include a thorough discussion of the absolute risk of recurrence in light of exact pathologic findings and postoperative PSA levels. As such, although adjuvant radiotherapy seems to be equally effective for all stages and postoperative PSA levels in terms of reducing the relative risk of recurrence, the absolute benefit is influenced by the absolute risk of recurrence. For men at low risk of recurrence, the benefits are modest. Moreover, all potential benefits must be weighed against the known risks.

\section{Recommendation 4}

Although the endorsement panel concurs, as noted in the discussion for guideline statement No. 3 and in our qualifying statement, not all men are at equal risk of recurrence. At this time, there are insufficient data to recommend different follow-up strategies based on differing risk of recurrence, and thus, all men should be observed after surgery.

\section{Recommendation 5}

Defining an exact cut point for PSA recurrence is challenging. We acknowledge this difficulty, and although we agree that $0.2 \mathrm{ng} / \mathrm{mL}$ is a reasonable cutoff and is widely used in research publications and in clinical practice, the benefits of using this cut point versus others are unclear. As such, we believe that the evidence to support this recommendation was clinical practice rather than level $\mathrm{C}$ evidence.

\section{Recommendation 6}

Although the endorsement panel agrees with this statement, the discussion centered on which imaging modalities to use. At this time, there is no clear consensus, and unfortunately, all imaging modalities have limited sensitivity and specificity in the low PSA range, where salvage radiotherapy is most effective (ie, PSA $<1$ $\mathrm{ng} / \mathrm{mL}$ ). We note that this is a rapidly evolving field with much research, and it is hoped that in the future, there will be more clarity on this point.

\section{Recommendation 7}

The word offer should be interpreted as analogous to the discussion regarding guideline statement No. 3 .

\section{Recommendation 8}

Although PSA control rates are best when salvage radiotherapy is administered when the PSA is $<1 \mathrm{ng} / \mathrm{mL}$, there is no guarantee of cure, because salvage radiotherapy is not curative for all men. There are many technical issues related to measuring the rate of PSA rise, typically measured as PSA doubling time (PSADT; ie, when to start counting, how many values, over what time period, and so on), ${ }^{5}$ but data suggest a short PSADT predicts a poor response to salvage radiotherapy in terms of PSA control. ${ }^{6}$ However, because these men are at the highest risk of clinically meaningful progression, ${ }^{7}$ they stand the most to gain from salvage radiotherapy, and indeed, retrospective data suggest that salvage radiotherapy improves overall survival, albeit only in those with a short PSADT. ${ }^{8}$
Finally, the ASCO endorsement panel added an additional qualifying statement not paired with a specific recommendation as follows:

Not all men who are candidates for adjuvant radiotherapy have the same risk of recurrence, and although radiotherapy is equally effective in terms of relative risk reduction, not all men will derive the same benefit. Items such as Gleason score (especially Gleason score 8 to 10), pathologic findings (especially seminal vesicle invasion or extensive positive margins), and an elevated postoperative PSA place men at particularly high risk of recurrence or clinical progression, and these men likely derive the greatest benefit in terms of absolute risk reduction from adjuvant radiotherapy. For other men, the absolute risk benefit is likely lower, resulting in a less favorable risk-benefit ratio. Similarly, not all men who develop a PSA recurrence have the same risk of clinically meaningful disease progression, and therefore, the risk-benefit ratio for salvage radiotherapy is likewise different for each patient.

The panel thought it was critical to highlight this point. As with the initial decision to undergo radical prostatectomy, deciding on adjuvant or salvage radiotherapy involves a consideration of the riskbenefit ratio. Although the risks are known, the benefits will vary for each patient based on his own risk of recurrence. Fortunately, this risk can be calculated using nomograms that incorporate not only preoperative and pathologic findings but also postoperative PSA values. ${ }^{9}$ Specifically, some men who are candidates for adjuvant radiotherapy based on entry criteria in reported clinical trials and an undetectable PSA may have favorable outcomes (ie, those with focal positive margins or low prostatectomy Gleason score), and for these men, the absolute benefits of adjuvant radiotherapy (compared with early salvage radiotherapy) may be low. Alternatively, men with more favorable pathology but a detectable postoperative PSA can be at high risk of clinically meaningful recurrence and thus stand to experience great benefits with adjuvant radiotherapy. Likewise, many men with a PSA recurrence, which can occur years after surgery when the patient has developed more comorbidities, have an indolent disease course. ${ }^{7}$ In these men, the benefits of salvage radiotherapy are likely low. Alternatively, others can experience an aggressive recurrence with a short PSADT, for which retrospective data suggest a survival advantage for salvage radiotherapy. ${ }^{8}$ Thus, there needs to be a risk-stratified approach to the discussion regarding the risk-benefit ratio of adjuvant and salvage radiotherapy.

\section{ENDORSEMENT RECOMMENDATION}

ASCO endorses the AUA/ASTRO guideline on adjuvant and salvage radiotherapy after prostatectomy by Thompson et $\mathrm{al}^{2}$ published in 2013 in Journal of Urology, with qualifying statements developed by the Endorsement Panel.

\section{ADDITIONAL RESOURCES}

More information, including a Data Supplement, a Methodology Supplement, slide sets, and clinical tools and resources, is available at www.asco.org/endorsements/adjuvantRTprostatectomy. Patient information is available at www.cancer.net. 


\section{AUTHORS' DISCLOSURES OF POTENTIAL CONFLICTS} OF INTEREST

Disclosures provided by the authors are available with this article at www.jco.org.

\section{REFERENCES}

1. Siegel R, Ma J, Zou Z, et al: Cancer statistics, 2014. CA Cancer J Clin 64:9-29, 2014

2. Thompson IM, Valicenti RK, Albertsen $P$, et al: Adjuvant and salvage radiotherapy after prostatectomy: AUA/ASTRO guideline. J Urol 190:441-449, 2013

3. Moreira DM, Presti JC Jr, Aronson WJ, et al: Natural history of persistently elevated prostate specific antigen after radical prostatectomy: Results from the SEARCH database. J Urol 182:2250-2255, 2009

\section{AUTHOR CONTRIBUTIONS}

Administrative support: R. Bryan Rumble

Manuscript writing: All authors

Final approval of manuscript: All authors
4. Abdollah F, Suardi N, Cozzarini C, et al: Selecting the optimal candidate for adjuvant radiotherapy after radical prostatectomy for prostate cancer: A long-term survival analysis. Eur Urol 63:998-1008, 2013

5. Arlen PM, Bianco F, Dahut WL, et al: Prostate specific antigen working group guidelines on prostate specific antigen doubling time. J Urol 179:21812185, 2008; discussion 2185-2186

6. Stephenson AJ, Shariat SF, Zelefsky MJ, et al: Salvage radiotherapy for recurrent prostate cancer after radical prostatectomy. JAMA 291:1325-1332, 2004

7. Freedland SJ, Humphreys EB, Mangold LA, et al: Risk of prostate cancer-specific mortality follow- ing biochemical recurrence after radical prostatectomy. JAMA 294:433-439, 2005

8. Trock BJ, Han M, Freedland SJ, et al: Prostate cancer-specific survival following salvage radiotherapy vs observation in men with biochemical recurrence after radical prostatectomy. JAMA 299: 2760-2769, 2008

9. Moreira DM, Presti JC Jr, Aronson WJ, et al: Postoperative prostate-specific antigen nadir improves accuracy for predicting biochemical recurrence after radical prostatectomy: Results from the Shared Equal Access Regional Cancer Hospital (SEARCH) and Duke Prostate Center databases. Int J Urol 17:914-922, 2010 


\section{AUTHORS' DISCLOSURES OF POTENTIAL CONFLICTS OF INTEREST}

Adjuvant and Salvage Radiotherapy After Prostatectomy: American Society of Clinical Oncology Clinical Practice Guideline Endorsement

The following represents disclosure information provided by authors of this manuscript. All relationships are considered compensated. Relationships are self-held unless noted. I = Immediate Family Member, Inst = My Institution. Relationships may not relate to the subject matter of this manuscript. For more information about ASCO's conflict of interest policy, please refer to www.asco.org/rwc or jco.ascopubs.org/site/ifc.

\section{Stephen J. Freedland}

Consulting or Advisory Role: Astellas Pharma, Medivation, Janssen

Biotech, Dendreon, Bayer

Speakers' Bureau: Dendreon

Research Funding: Dendreon (Inst), Bayer (Inst), Amgen (Inst), Janssen Biotech (Inst), Myriad Genetics (Inst), GenomeDx Biosciences (Inst), Mitomics (Inst), Metabolon (Inst), POM Wonderful (Inst)

Travel, Accommodations, Expenses: Sanofi, Myriad Genetics

\section{R. Bryan Rumble}

No relationship to disclose

\section{Antonio Finelli}

Stock or Other Ownership: Pfizer, Sanofi, Merck, Novo Nordisk, Bristol-Myers Squibb, Gilead Sciences, Abbvie, Actavis, Baxter International

Honoraria: Amgen, Janssen Oncology, Astellas Pharma

Consulting or Advisory Role: Amgen, Janssen Oncology, Astellas Pharma

\section{Ronald C. Chen \\ Research Funding: Accuray \\ Susan Slovin \\ Consulting or Advisory Role: Eisai}

Mark N. Stein

No relationship to disclose

\section{David S. Mendelson}

No relationship to disclose

\section{Colin Wackett}

No relationship to disclose

Howard M. Sandler

Consulting or Advisory Role: Medivation-Astellas, AstraZeneca, Janssen Pharmaceuticals, Bayer, Eviti

Research Funding: Myriad Genetics

Other Relationship: Caribou Publishing 


\section{Acknowledgment}

We thank the two Clinical Practice Guidelines Committee (CPGC) reviewers, Dana Rathkopf, MD, and Sandy Srinivas, MD, as well as the entire CPGC for their thoughtful reviews and insightful comments on this guideline endorsement. 